\title{
Aeroelastic Model Structure Computation for Envelope Expansion
}

\author{
Sunil L. Kukreja* \\ NASA Dryden Flight Research Center, Edwards, California, 93523-0273, USA
}

\begin{abstract}
Structure detection is a procedure for selecting a subset of candidate terms, from a full model description, that best describes the observed output. This is a necessary procedure to compute an efficient system description which may afford greater insight into the functionality of the system or a simpler controller design. Structure computation as a tool for black-box modelling may be of critical importance in the development of robust, parsimonious models for the flight-test community. Moreover, this approach may lead to efficient strategies for rapid envelope expansion which may save significant development time and costs.

In this study, a least absolute shrinkage and selection operator (LASSO) technique is investigated for computing efficient model descriptions of nonlinear aeroelastic systems. The LASSO minimises the residual sum of squares by the addition of an $\ell_{1}$ penalty term on the parameter vector of the traditional $\ell_{2}$ minimisation problem. Its use for structure detection is a natural extension of this constrained minimisation approach to pseudolinear regression problems which produces some model parameters that are exactly zero and, therefore, yields a parsimonious system description. Applicability of this technique for model structure computation for the F/A-18 Active Aeroelastic Wing using flight test data is shown for several flight conditions (Mach numbers) by identifying a parsimonious system description with a high percent fit for cross-validated data.
\end{abstract}

\section{Introduction}

S YSTEM identification, or black-box modelling, is a critical step in aircraft development, analysis and validation for S flight worthiness. The development and testing of aircraft typically takes many years and requires considerable expenditure of limited resources. One reason for lengthy development time/costs is inadequate knowledge of an appropriate model type or structure to use for parameter estimation. Selection of an insufficient model structure may lead to difficulties in parameter estimation, giving estimates with significant biases and/or large variances $!^{1}$ This often complicates control synthesis or renders it infeasible. The power of using structure detection techniques as a tool for model development (i.e. black-box modelling) is that it can provide a parsimonious system description which can describe complex aeroelastic behaviour over a large operating range. Consequently, this provides models that can be more robust and, therefore, reduce development time.

Moreover, when studying aeroelastic systems it may not be practical to assume that the exact model structure is well known a priori. In aerospace systems analysis one of the main objectives is not only to estimate system parameters but to gain insight into the structure of the underlying system. Therefore, structure computation is of significant relevance and importance to modelling \& design of aircraft and aerospace vehicles. Structure computation may indicate deficiencies in an analytical model and could lead to improved modelling strategies and also provide a parsimonious, black-box, system description for control synthesis. ${ }^{2}$

\section{NARMAX Model Form}

Recently, Kukreja and Brenne ${ }^{\sqrt{3}}$ showed that NARMAX (Nonlinear AutoRegressive, Moving Average eXogenous) identification is well suited to describing aeroelastic phenomena. The NARMAX structure is a general parametric form for modeling nonlinear systems. ${ }^{4}$ This structure describes both the stochastic and deterministic components of nonlinear systems. Many nonlinear systems are a special case of the general NARMAX structure.$^{[5}$ In this paper, we focus on a special class of NARMAX models; nonlinear polynomial models. The polynomial NARMAX structure

\footnotetext{
*Aerospace Research Engineer, Structural Dynamics Group, NASA Dryden Flight Research Center.
} 
models the input-output relationship as a nonlinear difference equation of the form

$$
z(n)=f^{l}\left[z(n-1), \cdots, z\left(n-n_{y}\right), u(n), \cdots, u\left(n-n_{u}\right), e(n-1), \cdots, e\left(n-n_{e}\right)\right]+e(n) .
$$

$f$ denotes a nonlinear mapping, $l$ is the order of the nonlinearity, $u$ is the controlled or exogenous input, $z$ is the measured output, and $e$ is the uncontrolled input or innovation. This nonlinear mapping may include a variety of nonlinear terms, such as terms raised to an integer power, products of present and past inputs, past outputs, or crossterms. In general, the nonlinear mapping, $f$, can be described by a wide variety of nonlinear functions such as sigmoids or splines. 6 This system description encompasses many forms of nonlinear difference equations that are linear-inthe-parameters.

Identifying a NARMAX model requires two things: (1) structure detection and (2) parameter estimation. Structure detection can be divided into: (1a) model order selection and (1b) selecting which parameters to include in the model. We consider model order selection as part of structure detection since, theoretically, there are an infinite number of candidate terms that could be considered initially. Establishing the model order, then, limits the choice of terms to be considered. For the NARMAX model, the system order is defined to be an ordered tuple as

$$
O \doteq\left[n_{u} n_{z} n_{e} l\right]
$$

where $n_{u}$ is the maximum lag on the input, $n_{z}$ the maximum lag on the output, $n_{e}$ the maximum lag on the error and $l$ is the maximum nonlinearity order. Note that for non-polynomial NARMAX models, $l$ may be simply replaced by a nonlinear mapping of some specified class. In this paper, we assume that the system order is known.

\section{Structure Detection}

The structure detection problem is that of selecting the subset of candidate terms that best describes the output. Therefore, the parametrisation of a system is still further reduced by determining which of the components are required. The maximum number of terms in a NARMAX model with $n_{z}, n_{u}$ and $n_{e}$ dynamic terms and $l$ th order nonlinearity is:

$$
p=\sum_{i=1}^{l} p_{i}+1 ; p_{i}=\frac{p_{i-1}\left(n_{u}+n_{z}+n_{e}+i\right)}{i}, p_{0}=1
$$

As a result, the number of candidate terms becomes very large for even moderately complex models making structure detection difficult. We define the maximum number of terms, $p$, as the number of candidate terms to be initially considered for identification. Due to the excessive parameterisation (the curse of dimensionality), the structure detection problem often leads to computationally intractable combinatorial optimisation problems.

\section{LASSO}

Recently, Kukreja et al ? $^{7}$ developed a novel approach to this computationally intractable combinatorial optimisation problem. The least absolute shrinkage and selection operator (LASSO) ${ }^{8}$ is least-squares like problem with the addition of an $\ell_{1}$ penalty on the parameter vector as

$$
\min _{\theta} \frac{1}{2}\|(\mathbf{Z}-\Phi \theta)\|_{2}^{2}+\lambda\|\theta\|_{1}
$$

where $\|\cdot\|_{2}$ denotes the $\ell_{2}$-norm, $\|\cdot\|_{1}$ denotes the $\ell_{1}$-norm, $\mathbf{Z} \in \mathbb{R}^{N \times 1}$ is a vector of outputs, $\Phi \in \mathbb{R}^{N \times p}$ is a matrix of regressors and $\theta \in \mathbb{R}^{p \times 1}$ is a vector of unknown system coefficients. Note that the regression matrix is a function of the measured input-outputs and unmeasured noise, which makes this a pseudolinear regression problem since $\Phi$ is (partly) unknown and must be estimated along with the parameters.

The regularisation parameter $\mathbb{R} \ni \lambda=\left[\lambda_{\min }, \ldots, \lambda_{\max }\right]$ controls the trade-off between approximation error and sparseness. LASSO shrinks the least-squares estimator (RHS Eqn. 4) towards 0 and potentially sets $\theta_{j}=0$ for some $j$. Consequently, LASSO behaves as a structure selection instrument.

Structure detection can provide useful process insights that can be used in subsequent development or refinement of physical models. Therefore, in the sequel, we investigate the applicability of LASSO to experimental aircraft data. Specifically, LASSO is used as a structure detection tool to assess whether the (i) underlying data is best described by a linear time-invariant (LTI) or nonlinear model and (ii) model structure is invariant during envelope expansion. 


\section{Experimental Aircraft Data}

The LASSO technique was assessed on experimental flight test data from the F/A-18 AAW project at NASA Dryden Flight Research Center. The data analysed for this study used collective aileron position input and structural accelerometer response output.

\section{A. Procedures}

Flight data was gathered during subsonic flutter clearance of the AAW. At each flight condition, the aircraft was subjected to band-limited white inputs, with uniform distribution and zero-mean. The inputs correspond to collective and differential aileron, collective and differential outboard leading edge flap, rudder, and collective stabilator excitations in the range of $\pm 0.5 \mathrm{rad}$ and $30 \mathrm{~Hz}$ bandwidth for $26 \mathrm{sec}$.

This paper considers accelerometer data measured during the collective aileron sweeps at Mach 0.85 and 0.95 both at $4,572 \mathrm{~m}(15,000 \mathrm{ft})$. The output was taken as the response of an accelerometer mounted near the wing leading edge just outside the wing fold (see Fig. 11). Data was sampled at $400 \mathrm{~Hz}$. For analysis, the recorded flight test data was

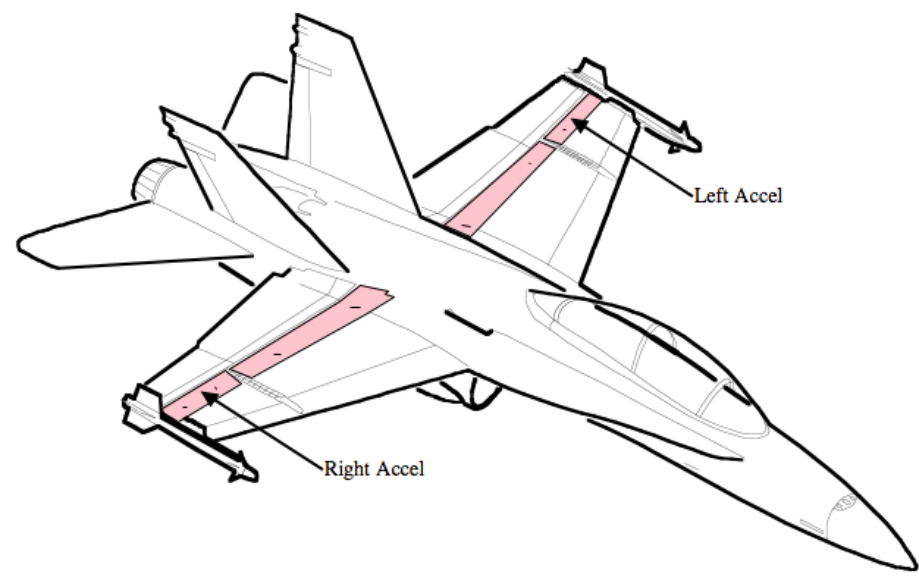

Figure 1. AAW accelerometer locations

decimated by a factor of 4 , resulting in a final sampling rate of $100 \mathrm{~Hz}$.

For identification the model posed for structure computation was an additive nonlinear model of the form

$$
z(n)=\sum_{v=1}^{q} \theta_{v} \varphi(n)+\sum_{w=1}^{r} \theta_{w} f(\varphi(n))+e(n) ; q+r=p .
$$

The regressors posed for this identification problem were of the form given in Eqn. 11, up to lag order 4, and the nonlinear mapping $f(\cdot)$, was chosen to be a hyperbolic tangent function $(\tanh (\cdot)) ; O=[444 \tanh ]$. It has been observed that aeroelastic structures present a well defined low frequency elastic mode composition $\sqrt{910}$ Therefore, the modal components are represented using a fourth-order dynamic expansion.11 Typically, an assumption is made that a structural nonlinearity exists in an aeroelastic system that affects not only flutter speed, but also the characteristics of the motion $\frac{1213}{13}$ An example of an aeroelastic nonlinearity is a preloaded spring tab system. It has been proposed that this type of nonlinearity be modelled as a saturator ${ }^{[13}$ In this paper, we chose to model this type of nonlinearity as a hyperbolic tangent, $\tanh (\cdot)$, because a wing section response typically saturates smoothly. This gave a full model description with 27 candidate terms.

The system was identified using scaled hyperbolic tangent functions because the input amplitude is less than \pm 1 (see Fig 2). The scale factors used for the input, output and error signals were in the range of $v=[0.11 .0]$ and increased in increments of 0.1 . A scaled hyperbolic tangent is denoted as $\tanh (\cdot, v)$. Models with every possible 
combination of scale factors were considered (i.e. structure computation was performed on 1,000 models). The model which yielded the highest cross-validation percent fit was deemed the best-fit model.

The system was identified applying the LASSO approach. For estimation, $N_{e}=2,570$ points were used from accelerometer response measurements on the left wing. For cross-validation, $N_{v}=2,570$ points were used from data collected at a similar location on the right wing. In both the estimation and cross-validation sets, the input was the same collective aileron position. The min-max regularisation parameter levels were set to $\lambda_{\min }=-10$ and $\lambda_{\max }=0.5$ $\left(10^{\lambda_{\min }} \leq \lambda \leq 10^{\lambda_{\max }}\right)$ with a discretisation grid of 1,000 logarithmically spaced $\lambda$ 's.

\section{B. Results}

The results of identifying the AAW data are presented. Fig. 2 shows the input-output trials used for this analysis. The
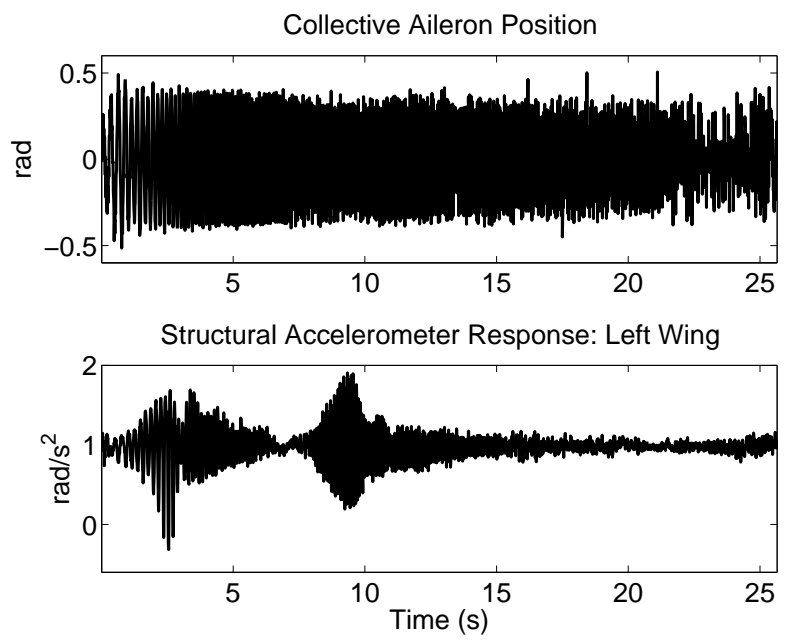

(a) Mach 0.85, Alt. 4,572 m (15,000 ft)

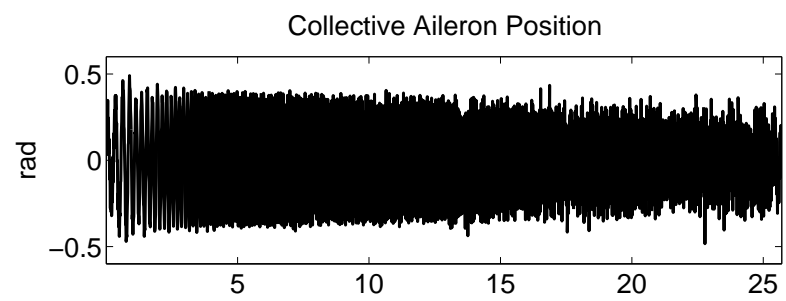

Structural Accelerometer Response: Left Wing

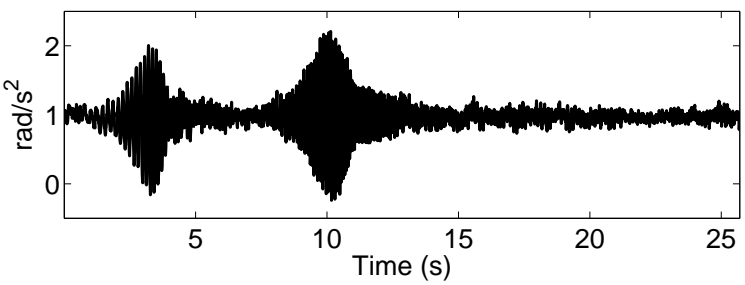

(b) Mach 0.95, Alt. 4,572 m (15,000 ft)

Figure 2. Estimation data. Upper panel: Recorded collective aileron position. Lower panel: Recorded structural accelerometer response.

data represents collective aileron position sequence and structural accelerometer response (left wing) used to compute the system structure.

Eqns. 6 \& 7 depict the model structure computed by the LASSO method

$$
\begin{aligned}
z(n) & =\hat{\theta}_{0}+\hat{\theta}_{1} u(n-1)+\hat{\theta}_{2} u(n-4)+\hat{\theta}_{3} \tanh (u(n-1), 0.4)+\hat{\theta}_{4} \tanh (u(n-2), 0.4) \\
& +\hat{\theta}_{5} \tanh (u(n-3), 0.4)+\hat{\theta}_{6} z(n-1)+\hat{\theta}_{7} z(n-2)+\hat{\theta}_{8} z(n-4)+\hat{\theta}_{9} \tanh (z(n-1), 0.4) \\
& +\hat{\theta}_{10} \tanh (z(n-3), 0.4)+\hat{\theta}_{11} \hat{\varepsilon}(n-1)+\hat{\theta}_{12} \hat{\varepsilon}(n-2)+\hat{\theta}_{13} \hat{\varepsilon}(n-4)+\hat{\theta}_{14} \tanh (\hat{\varepsilon}(n-1), 0.4) \\
& +\hat{\theta}_{15} \tanh (\hat{\varepsilon}(n-3), 0.4) \\
z(n)= & \hat{\vartheta}_{0}+\hat{\vartheta}_{1} u(n-1)+\hat{\vartheta}_{2} u(n-4)+\hat{\vartheta}_{3} \tanh (u(n-1), 0.4)+\hat{\vartheta}_{4} \tanh (u(n-2), 0.4)+\hat{\vartheta}_{5} z(n-1) \\
+ & \hat{\vartheta}_{6} z(n-2)+\hat{\vartheta}_{7} z(n-4)+\hat{\vartheta}_{8} \tanh (z(n-1), 0.4)+\hat{\vartheta}_{9} \tanh (z(n-3), 0.4)+\hat{\vartheta}_{10} \tanh (z(n-4), 0.4) \\
+ & \hat{\vartheta}_{11} \hat{\varepsilon}(n-1)+\hat{\vartheta}_{12} \hat{\varepsilon}(n-2)+\hat{\vartheta}_{13} \hat{\varepsilon}(n-4)+\hat{\vartheta}_{14} \tanh (\hat{\varepsilon}(n-1), 0.4)+\hat{\vartheta}_{15} \tanh (\hat{\varepsilon}(n-3), 0.4) \\
+ & \hat{\vartheta}_{16} \tanh (\hat{\varepsilon}(n-4), 0.4) .
\end{aligned}
$$

Eqn.6 6 represents the computed model structure for flight condition Mach 0.85 whilst Eqn.77represents the computed model structure for flight condition Mach 0.95. The computed model structures are represented by a combination of linear and nonlinear, lagged input-output terms and contain 15 and 16 terms for Mach 0.85 and 0.95 , respectively. Hence, the LASSO technique successfully produced a parsimonious model description from the full set of 27 candidate terms.

Fig. 3 shows the predicted output for a cross-validation data set for the identified structure ((a): Eqn.6. (b): Eqn. 7). The upper panel displays the full 26 s time history of the accelerometer response recorded on the right wing. The 
Structural Accelerometer Response: Right Wing

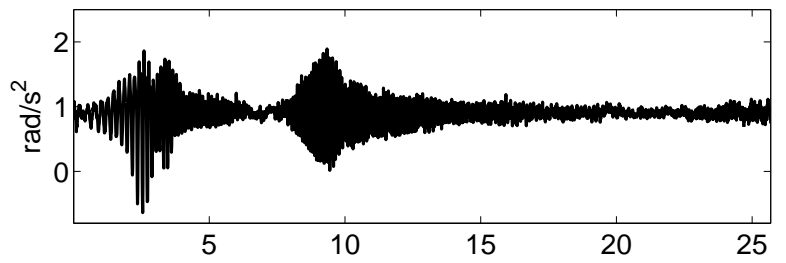

Cross-Validated Accelerometer Response (10s slice)

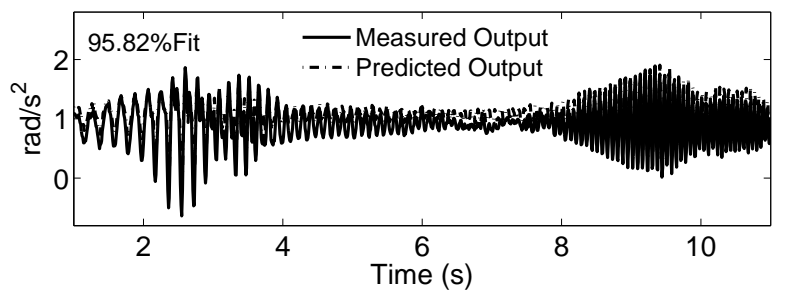

(a) Mach 0.85, Alt. 4,572 m (15,000 ft)
Structural Accelerometer Response: Right Wing

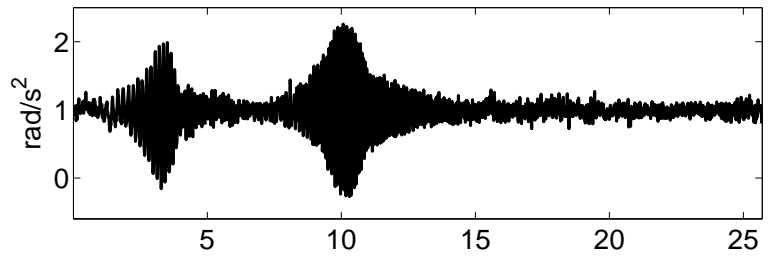

Cross-Validated Accelerometer Response (11s slice)

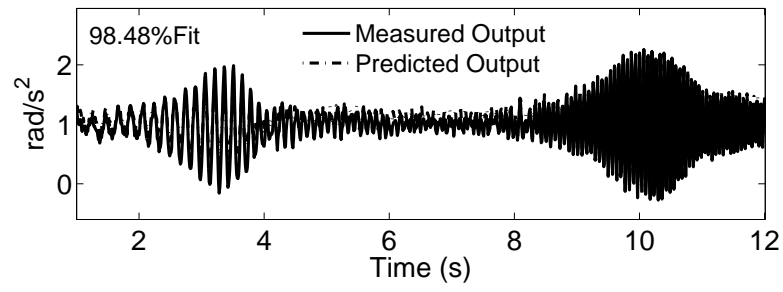

(b) Mach 0.95, Alt. 4,572 m (15,000 ft)

Figure 3. Cross-validation data. Upper panels: Full time history of structural accelerometer response. Lower panels: Predicted accelerometer response of right wing superimposed on top of measured accelerometer output.

lower panel displays a 10 (Mach 0.85) and 11 (Mach 0.95) second slice of the predicted output superimposed on top of the measured output. For Mach 0.85 (Fig.33(a)) the predicted output accounts for over $95 \%$ of the measured outputs variance whilst for Mach 0.95 (Fig. 3(b)) the predicted output accounts for over $98 \%$ of the measured outputs variance. The results demonstrate that the computed model structures are capable of reproducing the measured output with high accuracy.

\section{Discussion}

Experimental results demonstrate that structure computation as a tool for black-box modelling may be a useful tool for the analysis of dynamic aircraft data. The LASSO successfully reduced the number of regressors posed to aircraft aeroelastic data yielding a parsimonious model structure for each data set. Additionally, these parsimonious structures were capable of predicting a large portion of the cross-validation data, collected on the adjacent wing and with a different sensor. This suggests that the identified structures and parameters explain the data well. Using percent fit alone as an indicator of model goodness could lead to incorrect interpretations of model validity. However, in many cases, for nonlinear models this may be the only indicator that is readily available.

In this work, the results show that whilst the linear dynamics remained invariant for both flight conditions available for analysis, the nonlinear dynamics changed as Mach number increased. For Mach 0.85 the model (Eqn.6) displayed a richer nonlinear dynamic description associated with the input, possibly due to actuation stiction. When the Mach number was increased to 0.95 , with other flight conditions held invariant, the model (Eqn. 7) demonstrated a richer nonlinear dynamic description associated with the output, possibly associated with the ailerons. It is difficult to make definitive comments on the underlying physics responsible for this behaviour without extensive analysis of different flight conditions. The important points to note are, this study suggests (i) nonlinear models are appropriate to describe the dynamics behaviour of advanced aircraft and (ii) models describing aircraft dynamics vary with flight condition. This suggests nonlinear modelling may afford a robust and parsimonious system description over a larger operating regime and models used for prediction (e.g. control) are not invariant for all flight conditions. This may hold significant implications for aircraft development, saving time and money. + For this study, only hyperbolic tangent with fourth-order input, output and error lag was used as a basis function to explain the nonlinear behaviour of the F/A-18 AAW data. Clearly, different basis functions and a higher dynamic order (lag-order) should be investigated to determine if another basis can produce accurate model predictions with reduced complexity. Moreover, further studies are necessary to evaluate whether the model structure is invariant under different operating conditions, such as altitude, 
and model parameterisations.

This study illustrates the usefulness of structure detection as an approach to compute a parsimonious model of a highly complex nonlinear process, as demonstrated with experimental data of aircraft aeroelastic dynamics. Moreover, analysis of input-output data can provide useful process insights that can be used in subsequent development or refinement of physical models. In particular, morphological models are based on assumptions (e.g. these effects are important and those are negligible) which may be incorrect $14 \mid 15$ A structure computation approach to model identification may help uncover such surprises.

\section{Conclusions}

LASSO is a novel approach for detecting the structure of over-parameterised nonlinear models. These results may have practical significance in the analysis of aircraft dynamics during envelope expansion and could lead to more efficient control strategies. In addition, this technique could allow greater insight into the functionality of various systems dynamics, by providing a quantitative model which is easily interpretable.

\section{References}

\footnotetext{
${ }^{1}$ Ljung, L., System Identification: Theory for the User, Prentice Hall, Inc., Englewood Cliffs, New Jersey, 2nd ed., 1999.

${ }^{2}$ Harris, C. and Billings, S., Self Tuning and Adaptive Control: Theory and Applications, Peter Peregrinus, London, 2nd ed., 1985.

${ }^{3}$ Kukreja, S. and Brenner, M., "Nonlinear Aeroelastic System Identification with Application to Experimental Data," AIAA Journal of Guidance, Control, and Dynamics, Vol. 29, No. 2, 2006, pp. 374-381.

${ }^{4}$ Leontaritis, I. and Billings, S., "Input-output parametric models for non-linear systems Part I: deterministic non-linear systems," International Journal of Control, Vol. 41, No. 2, 1985, pp. 303-328.

${ }^{5}$ Chen, S. and Billings, S., "Representations of non-linear systems: the NARMAX model," International Journal of Control, Vol. 49, No. 3 , 1989, pp. 1013-1032.

${ }^{6}$ Billings, S. and Chen, S., "Extended Model Set, Global Data and Threshold Model Identification of Severely Non-linear Systems," International Journal of Control, Vol. 50, No. 5, 1989, pp. 1897-1923.

${ }^{7}$ Kukreja, S., Löfberg, J., and Brenner, M., "A Least Absolute Shrinkage and Selection Operator (LASSO) for Nonlinear System Identification," Proc. 14th IFAC Symp. System Identification, Vol. 14, Newcastle, Australia, March 2006, pp. 814-819.

${ }^{8}$ Tibshirani, R., "Regression Shrinkage and Selection via the LASSO," J. R. Statist. Soc. B, Vol. 58, No. 1, 1996, pp. 267288.

${ }^{9}$ Gawronski, W., Balanced Control of Flexible Structures, Springer-Verlag, London, 1st ed., 1996.

${ }^{10}$ Smith, R., "Eigenvalue Perturbation Models for Robust Control," IEEE Automatic Control, Vol. 40, No. 6, 1995, pp. 10631-1066.

${ }^{11}$ Baldelli, D., Chen, P. C., Liu, D. D., Lind, R., and Brenner, M., "Nonlinear Aeroelastic Modeling by Block-Oriented Identification," 45th AIAA/ASME/ASCE/AHS/ Structures, Structural Dynamics \& Materials Conference, Palm Springs, CA, 19-22 Apr. 2004 2004, pp. AIAA-20041938.

${ }^{12}$ Dowell, E., Edwards, J., and Strganac, T., "Nonlinear Aeroelasticity,” AIAA Journal of Aircraft, Vol. 40, No. 1, 2003 , pp. 857-874.

${ }^{13}$ Lee, B., Pricei, S., and Wong, Y., "Nonlinear aeroelastic analysis of airfoils: bifurcation and chaos," Progress in Aerospace Sciences, Vol. 35, No. 3, 1999, pp. 205-334.

${ }^{14}$ Pearson, R., “Nonlinear Input/Output Modelling," J. Proc. Cont., Vol. 5, No. 4, 1995, pp. 197-211.

${ }^{15}$ Pottmann, M. and Pearson, R., "Block-Oriented NARMAX Models with Output Multiplicities," AIChE Journal, Vol. 44, No. 1, 1998, pp. 131-140.
} 